\title{
Salud y condiciones de trabajo en trabajadores mayores
}

\section{Health and work conditions in older workers}

\section{Ordaz Castillo, Elena'; Ronda-Pérez, Elena ${ }^{2}$}

1. Escuela Nacional de Medicina del Trabajo. Instituto de Salud Carlos III. Madrid. España.

2. Centro de Investigación en Salud Laboral. Universitat Pompeu Fabra. Barcelona. España.

Recibido: 28-07-15

Aceptado: 22-09-15

\section{Correspondencia}

Elena Ordaz Castillo

Escuela Nacional de Medicina del Trabajo

Instituto de Salud Carlos III

Pabellón 13 Campus de Chamartín

Avda. Monforte de Lemos, N. ${ }^{\circ} 5$

28029 - Madrid. España.

Correo electrónico: eordaz@isciii.es

Teléfono: 918224025

Resumen

Introducción: En los últimos años, la población española ha experimentado un crecimiento acelerado de personas mayores. Las previsiones demográficas a corto-medio plazo describen un importante predominio de trabajadores mayores en el mercado laboral.

Objetivos: Identificar las diferencias según dos grupos de edad ( $<55$ años y $\geq 55$ años) en la percepción de las condiciones de trabajo y salud de la población trabajadora española.

Metodología: Las diferencias entre los dos grupos de edad se analizaron a partir de indicadores de condiciones de trabajo y de salud pertenecientes a la VII Encuesta Nacional de Condiciones de Trabajo del Instituto de Seguridad e Higiene en el Trabajo (VII_ENCT). El análisis consistió en el cálculo de las prevalencias y la odds ratio cruda-OR y ajustada por sexo con su correspondiente intervalo del confianza al $95 \%$

Resultados: El grupo de trabajadores más jóvenes presentan más riesgo de exposición a seis de los siete indicadores relacionados con las condiciones de trabajo (ruido, vibraciones, carga física, carga mental, autonomía y motivación). No obstante los trabajadores mayores tienen una peor percepción de su estado de salud $\left(\mathrm{OR}_{\mathrm{a}}=2,06[1,75-2,42]\right)$ y presentan en mayor medida problemas de salud que si bien les conducen a la visita médica más frecuentemente los relacionan menos con su actividad laboral.

Conclusiones: A tenor de los resultados, los trabajadores de 55 años y más refieren tener menos quejas respecto a sus condiciones laborales e incluso se sienten más autónomos y motivados. Es el deterioro físico y mental la principal limitación que encuentran estos trabajadores a la hora de ejercer sus tareas. Sería recomendable establecer políticas de promoción de la salud dentro de las empresas para mejorar los indicadores de salud y promover el envejecimiento activo de la población trabajadora española.

Med Segur Trab (Internet) 2015; 61 (240) 314-324

Palabras clave: Edad, lugares de trabajo, condiciones de trabajo, salud. 


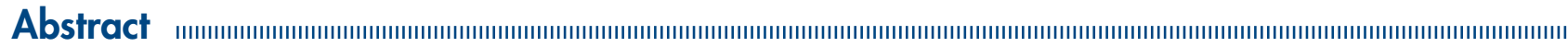

Introduction: In recent years, Spain's population has aged rapidly. Short and middle-term demographic forecasts describe a predominance of older workers in the labor market.

Objectives: To identify differences between two age groups (under 55 and aged 55 and over) in the perception of working conditions and health of the Spanish working population.

Metbodology: The differences between the two age groups were analyzed from work and health indicators belonging to the VII National Survey on Working Conditions, made by the Institute for Safety and Health at Work (VII_ENCT). The analysis consisted of calculating the prevalence and odds ratio raw and adjusted pro sex ratio- with its corresponding confidence interval of 95.

Results: The group of younger workers have showed a higher risk in six out of the seven indicators analyzed concerning working conditions (noise, vibration, physical and mental stress, autonomy and motivation). However, older workers have poorer perception of their health status (a OR = 2.06 [1.75 to 2.42]) and they have more health problems than younger workers, although less related to the working conditions.

Conclusions: According to the results, workers aged 55 and older report having fewer complaints about their working conditions and even feel more autonomous and motivated. The physical and mental deterioration is the main handicap for these workers when exercising their tasks. It would be advisable to establish policies to promote health within companies to improve health indicators and also to promote active aging in the Spanish working population.

Med Segur Trab (Internet) 2015; 61 (240) 314-324

Keywords: Aged, workplace, work conditions, bealth. 


\section{INTRODUCCIÓN}

La población europea envejece. Según declaraciones de la Comisión Europea, tres son las tendencias responsables de este cambio: 1) La continua subida de la esperanza de vida debido a una significativa mejora en la salud y calidad de vida de la población europea; 2) el aumento del grupo de edad de más de 65 años para el año 2030, momento en el cual la generación del baby boom alcanzará la edad de jubilación; y 3) el constante descenso del índice de natalidad por diversas razones.

En un informe publicado por el European Centre sobre envejecimiento las previsiones para el año 2025 de la población europea mayor a 50 años se sitúa por encima del 38\% casi 10 puntos porcentuales más que el año 2000ํ․ Este envejecimiento de la población lleva aparejado como consecuencia que la edad media de los trabajadores europeos sea más alta que nunca, con un claro predominio de los denominados, trabajadores mayores en el mercado laboral europeo.

En la UE-27 la tasa de empleo para las personas de 55-64 años aumentó del 36,9\% en el año 2000 al 46\% en el 2009 y las tendencias de la población activa en la UE-27 apuntan a que el grupo de edades de 55 a 64 años aumentará en un 16,2\% (9,9 millones) entre 2010 y 2030 mientras que el resto de grupos de edades mostrarán una tendencia a la baja, que va del 5,4\% (40-54 años) al 14,9\% (25-39 años). ${ }^{2,3}$

En nuestro país, según informó el INE en el documento "Proyecciones de Tasas de Actividad Globales y Específicas por Grupos de Edad y Sexo 2011-2026" ", se ha estimado que el volumen de la población activa de 55 años y más en el año 2026 será de más de 4 millones de trabajadores traducido a una tasa de crecimiento del 37,3\%, aunque es probable que está cifra sea mayor. Además resulta inquietante el descenso esperado del grupo de edad de entre 25 y 54 años, con un decrecimiento del $-7,2 \%$.

El proceso de envejecimiento lleva aparejado una serie de deficiencias en las funciones sensoriales, orgánicas y cognitivas. También se asocia con un incremento en la patología de carácter crónica y limitante tales como el cáncer o enfermedades cardiovasculares $^{5-7}$.

Atendiendo a las capacidades sensoriales, cabe destacar el deterioro auditivo ${ }^{8}$ y visual $^{9}$ con una reducción en la agudeza visual, campo visual, pérdida de capacidad de acomodación, discriminación del contraste y del color e incremento en la sensibilidad a deslumbramientos. La función cognitiva (la memoria, el razonamiento y la comprensión) puede empezar a deteriorarse a partir de los 45 años de edad, según diversos estudios ${ }^{10-11}$. A partir de la treintena, la capacidad cardiorrespiratoria y la fuerza muscular se reducen en un 1-2\% anualmente ${ }^{12}$ y a nivel respiratorio se produce además un deterioro progresivo de la función pulmonar ${ }^{13}$.

En el sistema músculo-esquelético, las personas pierden de 15 a $20 \%$ de su fuerza entre las edades de 20 a 60 años y, es frecuente encontrar pérdida de masa ósea, osteoporosis, pérdida de masa muscular, degeneración del cartílago articular y $\operatorname{artrosis}^{14}$.

El conjunto de estas limitaciones da lugar en muchas ocasiones a una cierta discriminación a la hora de contratar o en el trato con los compañeros. Así, los resultados del eurobarómetro $2012^{15}$, muestran que la discriminación por motivos de edad en el lugar de trabajo es la forma más frecuente de este tipo de discriminación. Aproximadamente 1 de cada 20 personas ha experimentado discriminación por motivos de edad en el lugar de trabajo (6\%) y 1 de cada 7 (1\%) ha sido testigo de este tipo de discriminación.

No obstante, aunque la salud y la capacidad física se deterioran a medida que envejecemos, existen otras destrezas y competencias especialmente las relacionadas con la comunicación organizacionales y habilidades sociales que solo maduran durante la segunda mitad de nuestra vida. El pensamiento estratégico, la agudeza de ingenio, la consideración, la sabiduría, la capacidad de deliberar, la capacidad de racionalizar, el control de la vida, la percepción global y las capacidades lingüísticas mejoran con la 
edad $^{2}$. Diversos estudios ${ }^{16-119}$ muestran que los trabajadores de más edad tienen más dedicación al trabajo, mejores relaciones sociales en el mismo y permanecen más tiempo en el puesto de trabajo que los jóvenes presentando a menudo tasas de absentismo más bajas $^{2,20}$.

Las habilidades, experiencia y madurez de los trabajadores de más edad compensan los posibles problemas de salud relacionados con la edad. Por otro lado, la edad parece que juega un papel protector frente a la siniestralidad laboral. Así se desprende del estudio de Guest (2014) ${ }^{21}$, donde los trabajadores menores de 30 años de edad tuvieron las tasas más altas de lesiones, con diferencias más significativas para las lesiones distintas de torceduras y esguinces que los mayores o, del Análisis del mercado laboral, condiciones de trabajo y siniestralidad ${ }^{22}$, en el que se refleja que la tasa de incidencia de los trabajadores más jóvenes (16-30 años) fue 1,3 veces mayor a la del grupo de trabajadores de edad intermedia ( 33-54 años ) intermedio y 1,6 superior a la de los mayores (< 55 y más).

El objetivo de este estudio ha sido identificar las diferencias en la distribución de los factores de riesgo laboral entre trabajadores mayores, considerados estos de 55 años y más y los más jóvenes ( $<55$ años).

\section{MATERIAL Y MÉTODOS}

La población de estudio fueron 7310 trabajadores asalariados entrevistados en la VII Encuesta Nacional de Condiciones de Trabajo del Instituto de Seguridad e Higiene en el Trabajo ${ }^{23}$.

La variable independiente grupo de edad, se generó a partir de la variable años cumplidos, distinguiendo entre trabajadores de 55 años y más y trabajadores menores de 55 años.

Como variables dependientes se analizaron variables relacionadas con las condiciones de trabajo, las características de la jornada de trabajo, conductas violentas, formación en seguridad y salud en el trabajo y el estado de salud.

Las condiciones de trabajo se midieron a través de siete indicadores: exposición a ruido, vibraciones, carga física, carga mental, apoyo social, motivación y autonomía. Alto nivel de ruido y vibraciones medido a través de una pregunta sobre la percepción del nivel de exposición. Para el resto de indicadores de este grupo, se realizó un sumatorio de las preguntas categóricas ordinales que conformaban cada ítem y se analizó la mediana. Se consideró alta exposición los valores por debajo o por encima de la mediana según fuera el orden de la escala de las variables categóricas ordinales

En cuanto a las características de la jornada de trabajo se ha tenido en cuenta el número de horas trabajadas por semana, el tipo de jornada, trabajar fines de semana y festivos y la conciliación laboral. Las conductas violentas en el trabajo se han analizado a partir de 12 preguntas de respuesta múltiple sobre los distintos tipos de violencia o discriminación. Los indicadores de salud se han medido a través de las preguntas sobre salud percibida, problemas de salud y accidentes de trabajo y, finalmente, la formación en materia de seguridad y salud en el trabajo.

\section{Análisis estadístico}

El análisis consistió en el cálculo de las prevalencias de cada uno de los indicadores por grupos de edad y la comparación del exceso de riesgo entre los dos grupos de edad creados mediante el cálculo de la odds ratio de prevalencia cruda-OR (trabajadores mayores frente a jóvenes) y ajustada pro sexo ( $\mathrm{OR}_{\mathrm{a}}$ ) con su correspondiente intervalo del confianza al 95\%. El programa estadístico utilizado fue el IBM SPSS Statics 22.0. 


\section{RESULTADOS}

Del total de asalariados, 915 son trabajadores de 55 años y más (12,5\%) y 6395 son trabajadores más jóvenes $(87,5 \%)$.

Las características sociodemográficas de los dos grupos de edad (tabla I) fueron similares en cuanto a sexo y a su distribución por sector de actividad no observándose diferencias estadísticamente significativas $(\mathrm{p}>0,005)$.

Tabla I. Características sociodemográficas de la muestra

\begin{tabular}{llccccc}
\hline & & \multicolumn{2}{c}{$\mathbf{1 7 - 5 4}$ años } & \multicolumn{2}{c}{55 y más años } & \multirow{2}{*}{ p-valor } \\
\cline { 3 - 6 } & & $\mathbf{n}$ & $\%$ & $\mathbf{n}$ & $\%$ & \\
\hline \multirow{2}{*}{ Sexo } & Hombres & 3.236 & 50,6 & 486 & 53,1 & $>0,05$ \\
& Mujeres & 3.159 & 49,4 & 429 & 46,9 & \\
\hline Sector & Agricultura & 209 & 3,3 & 23 & 2,5 & \\
actividad & Industria & 932 & 14,6 & 139 & 15,2 & \\
& Construcción & 452 & 7,1 & 58 & 6,3 & $<0,05$ \\
& Servicios & 4.801 & 75,1 & 695 & 76,0 & \\
& Total & 6.394 & 100 & 915 & 100 & \\
\hline
\end{tabular}

Base: total de trabajadores asalariados ENCT VII $(n=6395)$.

En cuanto a la distribución por las distintas actividades económicas se aprecia una mayor dispersión en los trabajadores más jóvenes mientras que el 35\% de los trabajadores mayores se concentran en Administración pública, educación y actividades sanitarias. (tabla II).

Tabla II. Distribución de la muestra en función de las actividades económicas (CNAE 2009) según grupos de edad (Datos en \%)

\begin{tabular}{lcc}
\hline & $\mathbf{1 7 - 5 4}$ años & $\mathbf{5 5}$ y más años \\
\hline Comercio al por menor, excepto de vehículos de motor & $9,6 \%$ & $4,3 \%$ \\
Administración Pública y defensa; Seguridad Social & $7,7 \%$ & $13,5 \%$ \\
Educación & $7,7 \%$ & $11,6 \%$ \\
Actividades sanitarias & $6,0 \%$ & $9,9 \%$ \\
Servicios de comidas y bebidas & $6,1 \%$ & $3,3 \%$ \\
Construcción de edificios & $4,4 \%$ & $3,3 \%$ \\
Actividades de los hogares & $3,3 \%$ & $5,5 \%$ \\
Comercio al por mayor e intermediarios del comercio & $3,0 \%$ & $2,5 \%$ \\
Otros servicios personales & $2,7 \%$ & $4,3 \%$ \\
Transporte terrestre y por tubería & $2,9 \%$ & $2,5 \%$ \\
Agricultura, ganadería, caza y servicios relac. con las mismas & $2,9 \%$ & $2,0 \%$ \\
Industria de la alimentación & $2,3 \%$ & $2,1 \%$ \\
Actividades de construcción especializada & $2,2 \%$ & $2,3 \%$ \\
Venta y reparación de vehículos de motor y motocicletas & $2,1 \%$ & $1,7 \%$ \\
Servicios financieros, excepto seguros y fondos de pensiones & $1,9 \%$ & $2,0 \%$ \\
\hline
\end{tabular}

Base: total de trabajadores asalariados. 


\section{Condiciones de trabajo}

Los trabajadores más jóvenes presentan una peor percepción de las condiciones de trabajo o más frecuencia de exposición a condiciones adversas para seis de los siete indicadores analizados (tabla III). En general, por debajo de los 55 años, los trabajadores manifiestan estar expuestos a niveles más altos de más ruido y vibraciones en el desempeño de sus tareas aunque estas diferencias no han resultado estadísticamente significativa para el caso del ruido y ligeramente significativas en el caso de las vibraciones ajustando por sexo.

Por otro lado, los más jóvenes presentan mayores exigencias físicas y mentales, gozan de menos autonomía y se encuentran menos motivados que los trabajadores mayores $(\mathrm{p}<0,000)$.

Son, sin embargo, los trabajadores más mayores quienes parecen contar con menos apoyo de sus jefes y sus compañeros a la hora de realizar su trabajo aunque esta diferencia no ha resultado estadísticamente significativa $(0,6 \%$ vs. 48,1\%, OR $=0,95-1,27)$.

Tabla III. Prevalencia y Odss de los indicadores de condiciones de trabajo por grupo de edad

\begin{tabular}{|c|c|c|c|c|c|c|c|c|c|c|}
\hline \multirow[b]{3}{*}{$\begin{array}{l}\text { Alta exposición } \\
\text { a Ruido }\end{array}$} & \multicolumn{2}{|c|}{ 17-54 años } & \multicolumn{2}{|c|}{55 y más años } & \multirow{3}{*}{$\begin{array}{r}\text { OR }_{c} \\
, 86\end{array}$} & \multirow{2}{*}{\multicolumn{2}{|c|}{ IC 95\% }} & \multirow{3}{*}{$\begin{array}{c}\mathrm{OR}_{\mathrm{a}} \\
, 84\end{array}$} & \multirow{2}{*}{\multicolumn{2}{|c|}{ IC 95\% }} \\
\hline & \multirow{2}{*}{$\begin{array}{c}\text { n } \\
707\end{array}$} & \multirow{2}{*}{$\begin{array}{c}\% \\
11,1\end{array}$} & \multirow{2}{*}{$\begin{array}{c}\text { n } \\
89\end{array}$} & \multirow{2}{*}{$\begin{array}{c}\% \\
9,8\end{array}$} & & & & & & \\
\hline & & & & & & ,68 & 1,08 & & ,66 & 1,06 \\
\hline $\begin{array}{l}\text { Alta exposición } \\
\text { a Vibraciones }\end{array}$ & 909 & 14,3 & 110 & 12,1 & ,82 & ,66 & 1,01 & ,78 & ,62 & ,96 \\
\hline $\begin{array}{l}\text { Altas exigencias } \\
\text { físicas }\end{array}$ & 2.697 & 46,3 & 344 & 41,0 &, 80 & ,69 & ,93 & ,80 & ,69 & ,93 \\
\hline $\begin{array}{l}\text { Altas exigencias } \\
\text { mentales }\end{array}$ & 3.085 & 50,4 & 371 & 42,1 &, 72 &, 62 & ,83 &, 72 & ,62 &, 83 \\
\hline $\begin{array}{l}\text { Falta Apoyo } \\
\text { social }\end{array}$ & 1.404 & 48,1 & 141 & 50,6 & 1,10 & ,96 & 1,27 & 1,10 & ,95 & 1,27 \\
\hline $\begin{array}{l}\text { Falta } \\
\text { Motivación }\end{array}$ & 2.707 & 47,5 & 376 & 40,8 & ,76 & ,66 & ,88 & ,76 & ,66 & ,89 \\
\hline Falta Autonomía & 2.938 & 51 & 160 & 44,7 &, 78 & ,67 & 90 & ,78 & ,67 & ,90 \\
\hline
\end{tabular}

$O R_{\mathrm{c}}=O R$ de trabajadores mayores respecto a jóvenes

$O R_{\mathrm{a}}=O R$ ajustada por sexo

\section{Horario de trabajo}

No se observan diferencias estadísticamente significativas en cuanto al número de horas trabajadas a la semana, 37,5 h el grupo de más edad y 37,9 horas los trabajadores más jóvenes $(\mathrm{p}=0,810)$. No obstante, el tipo de jornada también refleja una comportamiento desigual entre los dos grupos de edad ( $\mathrm{p}<0,005)$, mientras que casi la mitad de los trabajadores mayores realizan jornadas de trabajo continúas los más jóvenes ocupan más trabajos de jornada partida y a turnos (tabla IV).

Tabla IV. Tipo de jornada de trabajo según grupo de edad

\begin{tabular}{lccccc}
\hline & \multicolumn{2}{c}{$17-54$ años } & \multicolumn{2}{c}{55 y más años } & p-valor \\
\cline { 2 - 5 } & $\mathrm{n}$ & $\%$ & $\mathrm{n}$ & $\%$ & \\
\hline Jornada Partida & 2.299 & 36,8 & 290 & 32,2 & \\
Jornada Continua & 2.440 & 39,1 & 422 & 46,9 & \\
Rotativa/turnos & 1.505 & 24,1 & 188 & 20,9 & \\
Total & 6.244 & 100 & 900 & 100 & \\
\hline
\end{tabular}


La falta de conciliación, trabajar los sábados o tener que prolongar la jornada laboral son aspectos significativamente más prevalentes entre el grupo de trabajadores más jóvenes tal y como se refleja en la tabla V.

Tabla V. Prevalencia y Odds de indicadores de conciliación laboral según grupos de edad

\begin{tabular}{|c|c|c|c|c|c|c|c|c|c|c|}
\hline \multirow[b]{3}{*}{ Trabajar sábado } & \multicolumn{2}{|c|}{ 17-54 años } & \multicolumn{2}{|c|}{55 y más años } & \multirow{3}{*}{$\begin{array}{c}\text { ORc } \\
, 72\end{array}$} & \multirow{2}{*}{\multicolumn{2}{|c|}{ IC 95\% }} & \multirow{3}{*}{$\begin{array}{c}\mathbf{O R}_{\mathrm{a}} \\
, 73\end{array}$} & \multirow{2}{*}{\multicolumn{2}{|c|}{ IC 95\% }} \\
\hline & \multirow{2}{*}{$\frac{\mathrm{n}}{1.916}$} & \multirow{2}{*}{$\begin{array}{c}\% \\
30,1\end{array}$} & \multirow{2}{*}{$\begin{array}{c}\text { n } \\
217\end{array}$} & \multirow{2}{*}{$\frac{\%}{23,8}$} & & & & & & \\
\hline & & & & & & ,62 &, 85 & & ,62 & ,86 \\
\hline Trabajador domingo & 1.026 & 16,1 & 140 & 15,4 & ,94 &, 78 & 1,14 & ,95 &, 78 & 1,15 \\
\hline $\begin{array}{l}\text { Jornada laboral } \\
\text { prolongada }\end{array}$ & 1.481 & 23,3 & 177 & 19,6 & 80 & ,67 & ,95 &, 79 & ,66 & ,94 \\
\hline Falta conciliación & 1.404 & 22,0 & 141 & 15,4 & ,64 &, 53 &, 77 & ,64 &, 53 &, 78 \\
\hline
\end{tabular}

$O R_{\mathrm{c}}=O R$ de trabajadores mayores respecto a jóvenes

$O R_{\mathrm{a}}=$ OR ajustada por sexo

Las conductas discriminatorias afectan al 11,2\% de los trabajadores de menos edad frente al 9,2\% de los mayores. Cabe destacar las conductas discriminatorias por edad más prevalentes entre los más mayores (tabla VI).

Tabla VI: Prevalencia de conductas violentas por grupos de edad (\%)

\begin{tabular}{lcc}
\hline & $\mathbf{1 7 - 5 4}$ años & $\mathbf{5 5}$ y más años \\
\hline Amenazas de violencia física & 3,9 & 3,4 \\
Violencia física cometida por personas pertenecientes a su lugar de trabajo & 0,6 & 0,5 \\
Violencia física cometida por personas no pertenecientes a su lugar de & 2,4 & 2,2 \\
trabajo & & \\
Pretensiones sexuales no deseadas (acoso sexual) & 0,4 & 0,1 \\
Agresiones verbales, rumores o aislamiento social & 7,5 & 6,2 \\
Discriminación por la edad & 0,5 & 0,8 \\
Discriminación por la nacionalidad & 0,9 & 0,3 \\
Discriminación sexual/discriminación por género & 0,6 & 0,5 \\
Discriminación por la raza, origen étnico o color de su piel & 0,4 & 0,3 \\
Discriminación por la religión & 0,2 & 0,0 \\
Discriminación por una discapacidad & 0,1 & 0,1 \\
Discriminación por la orientación sexual & 0,1 & 0,0 \\
Ninguna & 88,8 & 90,8 \\
\hline
\end{tabular}

Base: 7310 trabajadores asalariados. Pregunta de respuesta múltiple.

\section{Formación}

La probabilidad de recibir formación entre los trabajadores mayores es menor que entre los jóvenes $\left(\mathrm{OR}_{\mathrm{a}}=0,85[0,73-0,98]\right)$.

Tabla VII. Prevalencia Odds de formación recibida según grupos de edad

\begin{tabular}{|c|c|c|c|c|c|c|c|c|}
\hline & \multicolumn{2}{|c|}{ 17-54 años } & \multicolumn{2}{|c|}{55 y más años } & \multirow{2}{*}{$\mathbf{O R}_{\mathrm{c}}$} & \multirow{2}{*}{ IC 95\% } & \multirow{2}{*}{$\mathbf{O R}_{\mathrm{a}}$} & \multirow{2}{*}{ IC 95\% } \\
\hline & $\mathbf{n}$ & $\%$ & $\mathbf{n}$ & $\%$ & & & & \\
\hline No & 2.442 & 38,6 & 381 & 42,3 & & & & \\
\hline $\mathrm{Si}$ & 3.882 & 61,4 & 520 & 57,7 & 0,86 & $0,75-0,99$ & 0,85 & $0,73-0,98$ \\
\hline Total & 6.324 & 100 & 901 & 100 & & & & \\
\hline
\end{tabular}

$O R_{\mathrm{c}}=O R$ de trabajadores mayores respecto a jóvenes

$O R_{\mathrm{a}}=$ OR ajustada por sexo 


\section{Estado de Salud}

En cuanto a la percepción del estado de salud (tabla VIII) los trabajadores de 55 años refieren peor su salud como regular/mala/muy mala 2,06 [1,75-2,42]).

Tabla VIII. Prevalencia y Odds de salud percibida según grupos de edad

\begin{tabular}{|c|c|c|c|c|c|c|c|c|}
\hline & \multicolumn{2}{|c|}{ 17-54 años } & \multicolumn{2}{|c|}{55 y más años } & \multirow{2}{*}{$\mathbf{O R}_{\mathrm{c}}$} & \multirow{2}{*}{ IC 95\% } & \multirow{2}{*}{$\mathbf{O R}_{\mathrm{a}}$} & \multirow{2}{*}{ IC 95\% } \\
\hline & $\mathbf{n}$ & $\%$ & $\mathbf{n}$ & $\%$ & & & & \\
\hline Regular/malo/muy malo & 1.023 & 16,0 & 254 & 27,7 & & & & \\
\hline Bueno/muy bueno & 5.367 & 84,0 & 662 & 72,3 & 2,01 & $1,71-2,36$ & 2,06 & $1,75-2,42$ \\
\hline Total & 6.390 & 100,0 & 916 & 100,0 & & & & \\
\hline
\end{tabular}

$O R_{c}=O R$ de trabajadores mayores respecto a jóvenes

$O R_{\mathrm{a}}=$ OR ajustada por sexo

En la tabla IX se resume la prevalencia de los problemas de salud de los trabajadores, la percepción de los mismos sobre su posible relación con el trabajo y las visitas médicas realizadas por estos problemas. En general, los trabajadores mayores de 55 años manifiestan tener más problemas de salud que el grupo más joven.

Destacan, los dolores osteomusculares (espalda, cuello y nuca, hombros, brazos y piernas), seguidos del cansancio o agotamiento, estrés o ansiedad y problemas visuales.

No obstante los trabajadores mayores asocian con menos frecuencia sus dolencias al trabajo aunque estas dolencias les llevan a efectuar más visitas al médico.

Tabla IX. Prevalencia de los problemas de salud por grupo de edad (datos en \%)

\begin{tabular}{|c|c|c|c|c|c|c|}
\hline & \multicolumn{3}{|c|}{ 17-54 años } & \multicolumn{3}{|c|}{55 y más años } \\
\hline & $\begin{array}{l}\text { Problemas } \\
\text { salud }\end{array}$ & $\begin{array}{c}\text { Agravado } \\
\text { producido } \\
\text { trabajo }\end{array}$ & $\begin{array}{l}\text { Visita } \\
\text { médica }\end{array}$ & $\begin{array}{l}\text { Problemas } \\
\text { salud }\end{array}$ & $\begin{array}{c}\text { Agravado } \\
\text { producido } \\
\text { trabajo }\end{array}$ & $\begin{array}{l}\text { Visita } \\
\text { médica }\end{array}$ \\
\hline Dolor de cuello/nuca & $32,0 \%$ & $87,5 \%$ & $57,0 \%$ & $36,4 \%$ & $87,4 \%$ & $69,8 \% * *$ \\
\hline Dolor de espalda & $50,0 \%$ & $88 \%$ & $58,4 \%$ & $57,6 \%$ & $85,6 \%$ & $73,3 \% * *$ \\
\hline Dolor en hombros, brazos & $26,3 \%$ & $88,3 \%$ & $56,6 \%$ & $34,7 \%$ & $85,2 \%$ & $75,6 \% * *$ \\
\hline Dolor en piernas, rodillas o pies & $21,3 \%$ & $81,8 \%$ & $52,9 \%$ & $28,3 \%$ & $75,0 \% *$ & $69,6 \%$ *** \\
\hline Problemas respiratorios & $3,9 \%$ & $33,6 \%$ & $79,8 \%$ & $4,8 \%$ & $47,7 \%$ & $71,4 \%$ \\
\hline Problemas de la voz & $4,8 \%$ & $73,8 \%$ & $57,9 \%$ & $5,8 \%$ & $69,8 \%$ & $56,8 \%$ \\
\hline Problemas de la piel & $3,7 \%$ & $38,2 \%$ & $67,0 \%$ & $4,5 \%$ & $43,9 \%$ & $72,2 \%$ \\
\hline Problemas auditivos (en los oídos) & $4,6 \%$ & $47,4 \%$ & $71,9 \%$ & $10,1 \%$ & $49,5 \%$ & $82,2 \%$ \\
\hline Problemas visuales (en los ojos) & $10,3 \%$ & $53,6 \%$ & $77,9 \%$ & $19,4 \%$ & $42,7 \% * * *$ & $86,9 \%$ \\
\hline Tensión arterial alta & $4,8 \%$ & $36,0 \%$ & $79,3 \%$ & $13,1 \%$ & $37,8 \%$ & $88,9 \%$ \\
\hline Dolor de cabeza & $14,5 \%$ & $60,9 \%$ & $48,3 \%$ & $13,4 \%$ & $60,2 \%$ & $67,6 \% * * *$ \\
\hline Problemas para conciliar el sueño & $9,2 \%$ & $69,1 \%$ & 46,3 & $14,0 \%$ & $60,9 \%$ & $59,7^{*}$ \\
\hline Estrés, ansiedad o nerviosismo & $16,5 \%$ & $83,5 \%$ & $44,2 \%$ & $19,7 \%$ & $75,0 \% * *$ & $55,6 \% \%$ \\
\hline Depresión o tristeza & $4,5 \%$ & $60,0 \%$ & $59,1 \%$ & $7,3 \%$ & $59,7 \%$ & $75,0 \%$ \\
\hline Cansancio, agotamiento & $18,3 \%$ & $88,2 \%$ & $29,8 \%$ & $21,4 \%$ & $83,2 \%$ & $45,4 \% * *$ \\
\hline Otros & $0,8 \%$ & $41,7 \%$ & $80,8 \%$ & $1,2 \%$ & $18,0 \%$ & $100 \%$ \\
\hline Ninguno & $29,1 \%$ & & & $16,1 \%$ & & \\
\hline
\end{tabular}

Base: Trabajadores que han señalado algún problema de salud). Datos en \%. Pregunta de respuesta múltiple;

$* P<0,005$

** $p=0,000$ 
Finalmente en la tabla $\mathrm{X}$ se refleja la prevalencia de accidentes de trabajo. Se observa un ligero incremento de los mismos en el grupo de más edad aunque la diferencia no ha resultado estadísticamente significativa $\left(O R_{a}=1,04[0,8-1,34]\right)$.

Tabla X. Prevalencia y odss de accidentes de trabajo según grupos de edad

\begin{tabular}{|c|c|c|c|c|c|c|c|c|}
\hline & \multicolumn{2}{|c|}{ 17-54 años } & \multicolumn{2}{|c|}{55 y más años } & \multirow{2}{*}{$\mathbf{O R}_{\mathrm{c}}$} & \multirow{2}{*}{ IC 95\% } & \multirow{2}{*}{$\mathbf{O R}_{\mathrm{a}}$} & \multirow{2}{*}{ IC 95\% } \\
\hline & n & $\%$ & n & $\%$ & & & & \\
\hline No & 5.891 & 92,2 & 840 & 91,9 & & & & \\
\hline Sí & 495 & 7,8 & 74 & 8,1 & 1,05 & $0,81-1,35$ & 1,04 & $0,80-1,34$ \\
\hline Total & 6.386 & 100 & 914 & 100 & & & & \\
\hline
\end{tabular}

\section{Discusión}

Los resultados de nuestro estudio reflejan una baja participación de los trabajadores mayores en el mercado laboral, con una representación del 14,2\% muy por debajo de la media europea señalada por Eurostat en el año 2013, donde la tasa de empleo de los trabajadores de 55 años y más alcanzó el 55,1\%

Posiblemente este resultado esté relacionado con la mayor dificultad de los trabajadores de más edad en el pasado reciente para competir con unos puestos de trabajo cada vez más escasos, en parte por su menor nivel educativo, las mayores dificultades de adaptación laboral como consecuencia de los cada vez más rápidos cambios tecnológicos, los mayores salarios que habría que pagarles y, finalmente, por las reticencias de los empresarios debidas a los mayores problemas de salud o accidentes laborales en que podrían incurrir.

La peor percepción del estado de salud de los trabajadores mayores, puesta de manifiesto en nuestro estudio, podría ser un indicativo de la mayor prevalencia de los problemas de salud evidenciados tal y como se refleja en numerosos hallazgos bibliográficos que describen el deterioro de las funcionas sensoriales y motoras asociadas al envejecimiento ${ }^{10-11 ; 24-27}$.

Padula $^{28}$, en su estudio diferencial sobre capacidades funcionales entre trabajadores mayores y jóvenes concluye que son los primeros quienes presentan una mayor prevalencia de enfermedades y consumo de medicamentos pero no se evidencian diferencias estadísticamente significativas en cuanto al índice de capacidad de trabajo (ICT) entre los dos grupos de edad. Similar resultado se desprende de la revisión sistemática llevada a cabo por Van der Berg $\&$ col $^{29}$, sobre los factores determinantes del Índice de capacidad de trabajo (ICT), en el que no se consigue establecer una relación directa con la edad y si con los factores individuales del trabajador, el estilo de vida, la condición física y las demandas del trabajo.

Las investigaciones científicas que evalúan el rendimiento profesional de los trabajadores mayores respecto a los más jóvenes, confirman «que no existe una diferencia significativa en el rendimiento laboral entre los trabajadores de mayor edad y los más jóvenes " ${ }^{30}$. Una explicación del alto rendimiento profesional que muestran los trabajadores mayores puede ser que estos pueden compensar cualquier deficiencia en sus competencias individuales con otras en las que sean más sobresalientes. Según $\mathrm{Ng}^{17}$, los trabajadores mayores utilizan diversas estrategias para adaptarse al deterioro de los recursos físicos que ayudan a hacer frente y mantener su funcionamiento en el lugar de trabajo por encima incluso de los trabajadores más jóvenes. La aptitud más positiva a la hora de valorar las condiciones de trabajo o la mayor satisfacción en su ejecución, resultados puestos de manifiesto en nuestro estudio, podrían constituir un ejemplo sobre estas estrategias de adaptación.

Por otro lado, la falta de significación estadística encontrada en nuestros resultados sobre la prevalencia de accidentes de trabajo coincide con los hallazgos del estudio de 
Guest, M \& col. ${ }^{21}$, cuyos resultados sostienen que los trabajadores mayores son más capaces de realizar su trabajo de forma segura, evitando así el riesgo y el accidente.

El contrapunto a todo lo expuesto, lo encontramos a la hora de analizar la formación complementaria en los trabajadores, herramienta imprescindible a la hora de actualizar sus cualificaciones y afianzar su puesto en el mercado laboral. La realidad, según se desprende del informe sobre Trabajo saludable en un Europa que envejece ${ }^{31}$ y de nuestros resultados es que los trabajadores de edad avanzada se forman mucho menos que otros grupos de edad.

Por tanto, el envejecimiento de la población trabajadora no debe verse como un problema y los trabajadores de más edad no deben constituir un riesgo para las empresas si no en mucho casos, una ventaja aprovechando de ellos su experiencia y su adaptabilidad al mercado laboral.

Se trataría entonces de apostar por políticas de promoción de la salud dentro de las empresas que apoyasen el desarrollo de hábitos de vida saludable, físicos y mentales y del fomento de la formación continuada entre los trabajadores más mayores ya que solo el aprendizaje puede preparar de verdad a los trabajadores del futuro hacia el cambio.

Una limitación de este estudio es el diseño transversal de la VII_ENCT que no permite establecer una relación causa efecto ya que los datos del evento y la exposición son medidas en el mismo momento.

Como conclusión, los trabajadores mayores perciben mejor las condiciones de trabajo a las que están expuestos manifestando soportar una menor carga física y mental respecto a los más jóvenes. En general, los principales problemas de salud están relacionados con el sistema musculoesquelético, el cansancio, el oído, la vista y el estrés si bien la prevalencia es mucho mayor en el grupo de más edad ocasionándoles un mayor número de visitas al médico. No obstante, los trabajadores de 55 años y más relacionan en menor medida estas dolencias con su actividad laboral. No se observan diferencias estadísticamente significativas en la prevalencia de accidentes de trabajo por grupos de edad.

\section{REFERENCIAS BIBLIOGRÁFICAS}

1. Asghar Zaidi. Features and Challenges of Population Ageing: The European Perspective. European Centre; 2008. Disponible en: http://www.euro.centre.org/data/1204800003_27721.pdf.

2. Promoción del envejecimiento activo. European Agency for Safety and Health at work. Disponible en: https://osha.europa.eu/es/tools-and-publications/publications/articles/promoting-active-ageing-in-theworkplace

3. Trabajadores de edad avanzada. Agencia Europea para la Seguridad y la Salud en el Trabajo 2015. Disponible en: https://osha.europa.eu/es/themes/osh-management-context-ageing-workforce

4. Proyecciones de Tasas de Actividad Globales y Específicas por Grupos de Edad y Sexo 2011-2026: Instituto Nacional de Estadística, 2011- Disponible en: http://www.ine.es/daco/daco42/daco4211/ proyecciones/epa_proyec_2011-2026.pdf

5. Grace Christ and Sadhna Diwan. Chronic Ilness and Aging. 2015.

6. Ruzicka $S$. The impact of normal aging processes and chronic illness on perioperative care of the elderly. Semin Perioper Nurs 1997 Jun 1;6(1):3-13.

7. Global Health and Aging. 2012. Word Healh Organization. Disponible en: http://www.who.int/ageing/ publications/global_health.pdf

8. NTP 366: Envejecimiento y trabajo: audición y motricidad. Instituto Nacional de Seguridad e Higiene en el Trabajo(INSHT). Disponible en http://www.insht.es/portal/site/Insht/

9. NTP 348: Envejecimiento y trabajo: la visión. Instituto Nacional de Seguridad e Higiene en el trabajo. Disponible en: http://www.insht.es/portal/site/Insht/

10. Sapkota RP, Van dL, I, Pardhan S. How does aging affect the types of error made in a visual short-term memory 'object-recall' task? Front Aging Neurosci 2014;6:346. 
11. Theppitak C, Lai V, Izumi H, Higuchi Y, Kumudini G, Movahed M, et al. Visual Recognition Memory Test Performance was Improved in Older Adults by Extending Encoding Time and Repeating Test Trials. J Occup Health 2015 Jan 19;56(6):453-60.

12. Stoltz, J,. F. Cardiovascular Biology: Endothelial Cell in Health and Hypertension, Volumen 1. In: J. F. Stoltz, editor. Netherlands: IOS Press; 2007.

13. Gulshan Sharma and James Goodwin. Effect of aging on respiratory system physiology and immunology. Clin Interv Aging 2006 Sep 1;1(3):253-60.

14. Deschenes MR1.. Effects of aging on muscle fibre type and size. Sports Med 2004;34(12):809-24.

15. European Comission. Active Ageing. Special Eurobarometer 378. 2012 Jan 1. (Consultado el 16/04/2015). Disponible en: http://ec.europa.eu/public_opinion/archives/ebs/ebs_378_en.pdf

16. Kirkham HS, Clark BL, Bolas CA, Lewis GH, Jackson AS, Fisher D, et al. Which modifiable health risks are associated with changes in productivity costs? Popul Health Manag 2015 Feb;18(1):30-8

17. Ng ES, Law A. Keeping up! Older workers' adaptation in the workplace after age 55. Can J Aging 2014 Mar;33(1):1-14.

18. Yeung DY, Fung HH, Chan DK. Positive effect of social work-related values on work outcomes: the moderating role of age and work situation. J Gerontol B Psychol Sci Soc Sci 2015 Mar;70(2):235-46.

19. Bohle P PCQM. Time to call it quits? The safety and health of older workers. Int J Health Serv 2010;40(1):2341.

20. Martocchio JJ1. Age-related differences in employee absenteeism: a meta-analysis. Psychol Aging 1989;4(4):409-14

21. Guest M, Boggess MM, Viljoen DA, Duke JM, Culvern CN. Age-related injury and compensation claim rates in heavy industry. Occup Med (Lond) 2014 Mar;64(2):95-103.

22. Análisis del mercado laboral, condiciones de trabajo y siniestrabilidad. Una perspectiva según la edad. Instituto Nacional de Seguridad e Higiene en el Trabajo. Disponible en: http://www.oect.es/ Observatorio/5\%20Estudios\%20tecnicos/Otros\%20estudios\%20tecnicos/Publicado/Ficheros/ INFORME\%20sobre\%20la\%20edad\%20\%28INSHT\%202013\%29.pdf

23. VII Encuesta Nacional de Condiciones de Trabajo. 2011. Instituto Nacional de Seguridad e Higiene en el Trabajo. Disponible en http://www.oect.es/InshtWeb/Contenidos/Documentacion/FICHAS\%20DE\%20 PUBLICACIONES/EN\%20CATALOGO/OBSERVATORIO/Informe\%20\%28VII\%20ENCT\%29.pdf

24. Norman JF, Adkins OC, Norman HF, Cox AG, Rogers CE. Aging and the visual perception of exocentric distance. Vision Res 2015 Apr;109(Pt A):52-58.

25. MacLeod KE, Satariano WA, Ragland DR. The Impact of Health Problems on Driving Status among Older Adults. J Transp Health 2014 Jun;1(2):86-94.

26. Smith P, Bielecky A, Ibrahim S, Mustard C, Saunders R, Beaton D, et al. Impact of pre-existing chronic conditions on age differences in sickness. absence after a musculoskeletal work injury: a path analysis approach. Scand J Work Environ Health 2014 Mar;40(2):167-75.

27. Jones MK, Latreille PL, Sloane PJ, Staneva AV. Work-related health risks in Europe: are older workers more vulnerable? Soc Sci Med 2013 Jul;88:18-29.

28. Padula RS, Comper ML, Moraes SA, Sabbagh C, Pagliato WJ, Perracini MR. The work ability index and functional capacity among older workers. Braz J Phys Ther 2013 Jul;17(4):382-91.

29. Van den Berg TI, Elders L, Zwart B, Burdorf A. The effects of work-related and individual factors onthe Work Ability Index: a systematic review. Occup Environ Med 2009;66:211-20.

30. Kruse A; Whal, HW. Personality development in old age. Z Gerontol Geriatr. 1999 Aug;32(4):279-93.

31. European Network for workplace health promotion. Trabajo Saludable en una Europa que Envejece. 2006. (Consultado 28/05/2015) Disponible en http://www.insht.es/PromocionSalud/Contenidos/ Promocion\%20Salud\%20Trabajo/Documentos\%20ENWHP/Guias/Ficheros/ENWHP_Ageing_Europe_ES. pdf 